\title{
Application of Hankel Transform for Solving a Fracture Problem of a Cracked Piezoelectric Strip Under Thermal Loading
}

\author{
Sei Ueda \\ Osaka Institute of Technology \\ Japan
}

\section{Introduction}

In this chapter, an example of the application of Hankel transform for solving a fracture problem will be explained. In discussing axisymmetric problems, it is advantageous to use polar coordinates, and the Hankel transform method is powerful to solve the general equations in polar coordinates. A brief account of the Hankel transform will be given. Here $f$ is a function of $r$, its transform is indicated by a capital $F, J_{v}$ is the $v$ th order Bessel function of the first kind, and the nature of the transformation either by a suffix or by a characteristic new variable $s$. It will be assumed without comment that the integrals in question exist, and that, if necessary, the functions and their derivatives tend to zero as the variable tends to infinity. The Hankel transform of order $v>-1 / 2, H_{v}[f(r)]$ or $F_{v}(s)$, of a function $f(r)$ is defined as

$$
H_{v}[f(r)] \equiv F_{v}(s)=\int_{0}^{\infty} r J_{v}(s r) f(r) d r
$$

and its inversion formula is

$$
f(r)=\int_{0}^{\infty} s J_{v}(s r) F_{v}(s) d s
$$

Also, integrating by parts twice gives

$$
H_{v}\left[\frac{d^{2} f}{d r^{2}}+\frac{1}{r} \frac{d f}{d r}-\frac{v^{2}}{r^{2}} f\right]=-s^{2} F_{v}(s)
$$

provided that $r f(r)$ and $r d f(r) / d r$ tend to zero as $r \rightarrow 0$ and as $r \rightarrow \infty$.

The piezoelectric materials have attracted considerable attention recently. Owing to the coupling effect between the thermo-elastic and electric fields in piezoelectric materials, thermo-mechanical disturbances can be determined form measurement of the induced electric potential, and the ensuing response can be controlled through application of an appropriate electric field (Rao \& Sunar, 1994). For successful and efficient utilization of 
piezoelectric as sensors and actuators in intelligent systems, several researches on piezothermo-elastic behavior have been reported (Tauchert, 1992).

Moreover a better understanding of the mechanics of fracture in piezoelectric materials under thermal load conditions is needed for the requirements of reliability and lifetime of these systems. Using the Fourier transform, the present author studied the thermally induced fracture of a piezoelectric strip with a two-dimensional crack (Ueda, 2006a, 2006b).

Here the mixed-mode thermo-electro-mechanical fracture problem for a piezoelectric material strip with a penny-shaped crack is considered. It is assumed that the strip is under the thermal loading. The crack faces are supposed to be insulated thermally and electrically. By using the Hankel transform (Sneddon \& Lowengrub, 1969), the thermal and electromechanical problems are reduced to a singular integral equation and a system of singular integral equations (Erdogan \& Wu, 1996), respectively, which are solved numerically (Sih, 1972). Numerical calculations are carried out, and detailed results are presented to illustrate the influence of the crack size and the crack location on the stress and electric displacement intensity factors. The temperature, stress and electric displacement distributions are also presented.

\section{Formulation of the problem}

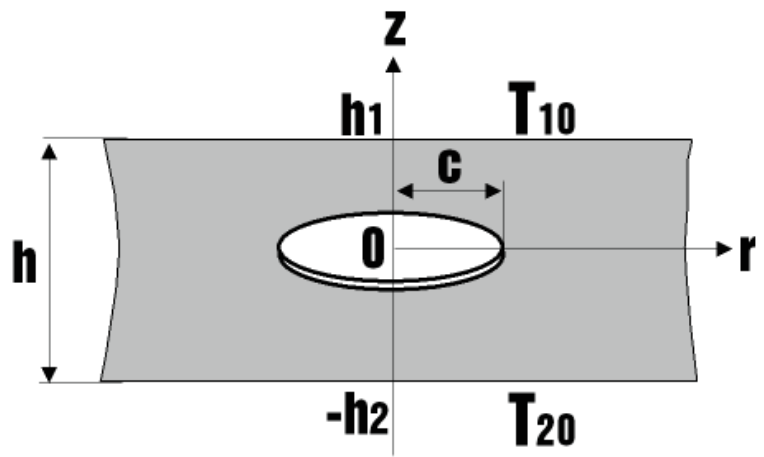

Fig. 1. Penny-shaped crack in a piezoelectric strip

A penny-shaped crack of radius $c$ is embedded in an infinite long piezoelectric strip of thickness $h=h_{1}+h_{2}$ as shown in Figure 1. The crack is located parallel to the boundaries and at an arbitrary position in the strip, and the crack faces are supposed to be insulated thermally and electrically. The cylindrical coordinate system is denoted by $(r, \theta, z)$ with its origin at the center of the crack face and the plane $r-\theta$ along the crack plane, where $z$ is the poling axis. It is assumed that uniform temperatures $T_{10}$ and $T_{20}$ are maintained over the stress-free boundaries. In the following, the subscripts $r, \theta, z$ will be used to refer to the direction of coordinates. The material properties, such as the elastic stiffness constants, the piezoelectric constants, the dielectric constants, the stress-temperature coefficients, the coefficients of heat conduction, and the pyroelectric constant, are denoted by $c_{k l}, e_{k l}, \varepsilon_{k k}$, $\lambda_{k k}(k, l=1,2,3), \kappa_{r}, \kappa_{z}$, and $p_{z}$, respectively.

The constitutive equations for the elastic field are 


$$
\left.\begin{array}{c}
\sigma_{r r i}=c_{11} \frac{\partial u_{r i}}{\partial r}+c_{12} \frac{u_{r i}}{r}+c_{13} \frac{\partial u_{z i}}{\partial z}+e_{31} \frac{\partial \phi_{i}}{\partial z}-\lambda_{11} T_{i}, \\
\sigma_{\theta \theta i}=c_{12} \frac{\partial u_{r i}}{\partial r}+c_{11} \frac{u_{r i}}{r}+c_{13} \frac{\partial u_{z i}}{\partial z}+e_{31} \frac{\partial \phi_{i}}{\partial z}-\lambda_{11} T_{i}, \\
\sigma_{z z i}=c_{13} \frac{\partial u_{r i}}{\partial r}+c_{13} \frac{u_{r i}}{r}+c_{33} \frac{\partial u_{z i}}{\partial z}+e_{33} \frac{\partial \phi_{i}}{\partial z}-\lambda_{33} T_{i}, \\
\sigma_{z r i}=c_{44}\left(\frac{\partial u_{z i}}{\partial r}+\frac{\partial u_{r i}}{\partial z}\right)+e_{15} \frac{\partial \phi_{i}}{\partial r}
\end{array}\right\}
$$

where $T_{i}(r, z)$ is the temperature, $\phi_{i}(r, z)$ is the electric potential, $u_{r i}(r, z), u_{z i}(r, z)$ are the displacement components, $\sigma_{r r i}(r, z), \sigma_{\theta \theta i}(r, z), \sigma_{z z i}(r, z), \sigma_{z r i}(r, z)(i=1,2)$ are the stress components. The subscript $i=1,2$ denotes the thermo-electro-elastic fields in $0 \leq z \leq h_{1}$ and $-h_{2} \leq z \leq 0$, respectively. For the electric field, the constitutive relations are

$$
\left.\begin{array}{c}
D_{r i}=e_{15}\left(\frac{\partial u_{z i}}{\partial r}+\frac{\partial u_{r i}}{\partial z}\right)-\varepsilon_{11} \frac{\partial \phi_{i}}{\partial r} \\
e_{31} \frac{\partial u_{r i}}{\partial r}+e_{31} \frac{u_{r i}}{r}+e_{33} \frac{\partial u_{z i}}{\partial z}-\varepsilon_{33} \frac{\partial \phi_{i}}{\partial z}+p_{z} T_{i}
\end{array}\right\} \quad(i=1,2)
$$

where $D_{r i}(r, z), D_{z i}(r, z)(i=1,2)$ are the electric displacement components.

The governing equations for the thermo-electro-elastic fields of the medium may be expressed as follows:

$$
\begin{gathered}
\kappa^{2}\left(\frac{\partial^{2} T_{i}}{\partial r^{2}}+\frac{1}{r} \frac{\partial T_{i}}{\partial r}\right)+\frac{\partial^{2} T_{i}}{\partial z^{2}}=0 \quad(i=1,2) \\
c_{11}\left(\frac{\partial^{2} u_{r i}}{\partial r^{2}}+\frac{1}{r} \frac{\partial u_{r i}}{\partial r}-\frac{\partial u_{r i}}{r^{2}}\right)+c_{44} \frac{\partial^{2} u_{r i}}{\partial z^{2}}+\left(c_{13}+c_{44}\right) \frac{\partial^{2} u_{z i}}{\partial r \partial z}+\left(e_{31}+e_{15}\right) \frac{\partial^{2} \phi_{i}}{\partial r \partial z}=\lambda_{11} \frac{\partial T_{i}}{\partial r}, \\
\left.c_{44}\left(\frac{\partial^{2} u_{z i}}{\partial r^{2}}+\frac{1}{r} \frac{\partial u_{z i}}{\partial r}\right)+c_{33} \frac{\partial^{2} u_{z i}}{\partial z^{2}}+\left(c_{13}+c_{44}\right)\left(\frac{\partial^{2} u_{r i}}{\partial r \partial z}+\frac{1}{r} \frac{\partial u_{r i}}{\partial z}\right)+e_{15}\left(\frac{\partial^{2} \phi_{i}}{\partial r^{2}}+\frac{1}{r} \frac{\partial \phi_{i}}{\partial r}\right)+e_{33} \frac{\partial^{2} \phi_{i}}{\partial z^{2}}=\lambda_{33} \frac{\partial T_{i}}{\partial z},\right)_{(i=1,2)}\left(\frac{\partial^{2} u_{z i}}{\partial r^{2}}+\frac{1}{r} \frac{\partial u_{z i}}{\partial r}\right)+e_{33} \frac{\partial^{2} u_{z i}}{\partial z^{2}}+\left(e_{15}+e_{31}\right)\left(\frac{\partial^{2} u_{r i}}{\partial r \partial z}+\frac{1}{r} \frac{\partial u_{r i}}{\partial z}\right)-\varepsilon_{11}\left(\frac{\partial^{2} \phi_{i}}{\partial r^{2}}+\frac{1}{r} \frac{\partial \phi_{i}}{\partial r}\right)-\varepsilon_{33} \frac{\partial^{2} \phi_{i}}{\partial z^{2}}=-p_{z} \frac{\partial T_{i}}{\partial z}
\end{gathered}
$$

where $\kappa^{2}=\kappa_{r} / \kappa_{z}$.

The boundary conditions can be written as

$$
\left.\begin{array}{cc}
\frac{\partial}{\partial z} T_{1}(r, 0)=0 & (0 \leq r<c) \\
T_{1}(r, 0)=T_{2}(r, 0) & (c \leq r<\infty)
\end{array}\right\}
$$




$$
\left.\begin{array}{c}
T_{1}\left(r, h_{1}\right)=T_{10}, \\
\frac{\partial}{\partial z} T_{1}(r, 0)=\frac{\partial}{\partial z} T_{2}(r, 0), \\
T_{2}\left(r,-h_{2}\right)=T_{20}
\end{array}\right\} \quad(0 \leq r<\infty)
$$

for thermal loading conditions and

$$
\begin{aligned}
& \left.\sigma_{z z 1}(r, 0)=0 \quad(0 \leq r<c)\right\} \\
& \left.u_{z 1}(r, 0)=u_{z 2}(r, 0) \quad(c \leq r<\infty)\right\} \\
& \left.\sigma_{z r 1}(r, 0)=0 \quad(0 \leq r<c)\right\} \\
& \left.u_{r 1}(r, 0)=u_{r 2}(r, 0) \quad(c \leq r<\infty)\right\} \\
& \left.D_{z 1}(r, 0)=0 \quad(0 \leq r<c)\right\} \\
& \left.\phi_{1}(r, 0)=\phi_{2}(r, 0) \quad(c \leq r<\infty)\right\} \\
& \sigma_{z z 1}(r, 0)=\sigma_{z z 2}(r, 0), \quad \sigma_{z z 1}\left(r, h_{1}\right)=0, \quad \sigma_{z z 2}\left(r,-h_{2}\right)=0, \\
& \sigma_{z r 1}(r, 0)=\sigma_{z r 2}(r, 0), \quad \sigma_{z r 1}\left(r, h_{1}\right)=0, \quad \sigma_{z r 2}\left(r,-h_{2}\right)=0, \quad(0 \leq r<\infty) \\
& D_{z 1}(r, 0)=D_{z 2}(r, 0), \quad D_{z 1}\left(r, h_{1}\right)=0, \quad D_{z 2}\left(r,-h_{2}\right)=0
\end{aligned}
$$

for the electromechanical conditions.

\section{Temperature field}

For the problem considered here, it is convenient to represent the temperature as the sum of two functions.

$$
T_{i}(r, z)=T^{(1)}(z)+T_{i}^{(2)}(r, z) \quad(i=1,2)
$$

where $T^{(1)}(z)$ satisfies the following equation and boundary conditions:

$$
\left.\begin{array}{c}
\frac{d^{2} T^{(1)}}{d z^{2}}=0 \\
T^{(1)}\left(h_{1}\right)=T_{10}, \\
T^{(1)}\left(-h_{2}\right)=T_{20}
\end{array}\right\}
$$

and $T_{i}^{(2)}(r, z)(i=1,2)$ is subjected to the relations:

$$
\left.\begin{array}{c}
\kappa^{2}\left(\frac{\partial^{2} T_{i}^{(2)}}{\partial r^{2}}+\frac{1}{r} \frac{\partial T_{i}^{(2)}}{\partial r}\right)+\frac{\partial^{2} T_{i}^{(2)}}{\partial z^{2}}=0 \quad(i=1,2) \\
\frac{\partial}{\partial z} T_{1}^{(2)}(r, 0)=-\frac{d}{d z} T^{(1)}(0) \quad(0 \leq r<c) \\
T_{1}^{(2)}(r, 0)=T_{2}^{(2)}(r, 0) \quad(c \leq r<\infty)
\end{array}\right\}
$$




$$
\left.\begin{array}{c}
T_{1}^{(2)}\left(r, h_{1}\right)=0 \\
\frac{\partial}{\partial z} T_{1}^{(2)}(r, 0)=\frac{\partial}{\partial z} T_{2}^{(2)}(r, 0), \\
T_{2}^{(2)}\left(r,-h_{2}\right)=0
\end{array}\right\} \quad(0 \leq r<\infty)
$$

It is easy to find from Eqs.(12) and (13) that

$$
T^{(1)}(z)=\frac{1}{h_{1}+h_{2}}\left\{\left(T_{10}-T_{20}\right) z+T_{10} h_{2}+T_{20} h_{1}\right\}
$$

By applying the Hankel transform to Eq.(14) (Sneddon \& Lowengrub, 1969), we have

$$
T_{i}^{(2)}(r, z)=\sum_{j=1}^{2} \int_{0}^{\infty} D_{i j}(s) J_{0}(s r) \exp \left(s \tau_{i j} z\right) d s \quad(i=1,2)
$$

where $D_{i j}(s)(i, j=1,2)$ are unknown functions to be solved and $\tau_{i j}(i, j=1,2)$ are given by

$$
\left.\begin{array}{c}
\tau_{11}=\tau_{22}=-\kappa, \\
\tau_{12}=\tau_{21}=\kappa
\end{array}\right\}
$$

Taking the second boundary condition (15) into consideration, the problem may be reduced to a singular integral equation by defining the following new unknown function $G_{0}(r)$ (Erdogan \& Wu, 1996):

$$
G_{0}(r)=\left\{\begin{array}{cc}
\frac{\partial}{\partial r}\left\{T_{1}^{(2)}(r, 0)-T_{2}^{(2)}(r, 0)\right\} & (0 \leq r<c) \\
0 & (c \leq r<\infty)
\end{array}\right\}
$$

Making use of the first boundary condition (15) with Eqs.(16), we have the following singular integral equation for the determination of the unknown function $G_{0}(t)$ :

$$
\int_{0}^{c} t\left\{M_{0}^{(1)}(t, r)+M_{0}^{(2)}(t, r)\right\} G_{0}(t) d t=-\frac{2}{\kappa} \frac{T_{10}-T_{20}}{h_{1}+h_{2}} \quad(0 \leq r<c)
$$

In Eq.(21), the kernel functions $M_{0}^{(1)}(t, r)$ and $M_{0}^{(2)}(t, r)$ are given by

$$
\begin{gathered}
M_{0}^{(1)}(t, r)=\left\{\begin{array}{cc}
\frac{2}{\pi} \frac{1}{t^{2}-r^{2}} E\left(\frac{r}{t}\right) & (r<t), \\
\frac{2}{\pi}\left\{\frac{r}{t\left(t^{2}-r^{2}\right)} E\left(\frac{t}{r}\right)+\frac{1}{r t} K\left(\frac{t}{r}\right)\right\} & (r>t)
\end{array}\right\} \\
M_{0}^{(2)}(t, r)=-\int_{0}^{\infty} s\left\{\frac{2 \rho_{1}(s) \rho_{2}(s)}{\kappa \rho_{0}(s)}+1\right\} J_{0}(s r) J_{1}(s t) d s
\end{gathered}
$$

where $K$ and $E$ are complete elliptic integrals of the first and second kind, and $\rho_{k}(s)(k=0,1,2)$ are given by 


$$
\left.\begin{array}{c}
\rho_{0}(s)=\rho_{2}(s)\left\{1-\exp \left(-2 s \kappa h_{1}\right)\right\}-\rho_{1}(s)\left\{1-\exp \left(-2 s \kappa h_{2}\right)\right\}, \\
\rho_{i}(s)=\tau_{i 1}-\tau_{i 2} \exp \left(-2 s \kappa h_{i}\right) \quad(i=1,2)
\end{array}\right\}
$$

Once $G_{0}(t)$ is obtained from Eq.(21), the temperature field can be easily calculated as follows:

$$
T_{i}^{(2)}(r, z)=\sum_{j=1}^{2} T_{i j}^{(2)}(r, z) \quad(i=1,2)
$$

where

$$
T_{i j}^{(2)}(r, z)=\int_{0}^{\infty} R_{i j}(s) R_{0}(s) J_{0}(s r) \exp \left(s \tau_{i j} z\right) d s \quad(i, j=1,2)
$$

with

$$
\left.\begin{array}{rr}
R_{0}(s)=\int_{0}^{c} t G_{0}(t) J_{1}(s t) d t, & \\
R_{11}(s)=-\frac{\rho_{2}(s)}{\rho_{0}(s)}, & R_{12}(s)=\frac{\rho_{2}(s)}{\rho_{0}(s)} \exp \left(-2 s \kappa h_{1}\right), \\
R_{21}(s)=-\frac{\rho_{1}(s)}{\rho_{0}(s)}, & R_{22}(s)=\frac{\rho_{1}(s)}{\rho_{0}(s)} \exp \left(-2 s \kappa h_{2}\right)
\end{array}\right\}
$$

On the plane $z=0$, the temperatures $T_{i}^{(2)}(r, 0)(i=1,2)$ are reduced to

$$
T_{i}^{(2)}(r, 0)=\frac{(-1)^{i}}{2} \int_{r}^{c} G_{0}(t) d t+\int_{0}^{\infty}\left\{\sum_{j=1}^{2} R_{i j}(s)-\frac{(-1)^{i}}{2}\right\} R_{0}(s) J_{0}(s r) d s \quad(i=1,2)
$$

\section{Thermally induced elastic and electric fields}

The non-disturbed temperature filed $T^{(1)}(z)$ given by Eq.(17) does not induce the stress and electric displacement components, which affect the singular field. Thus, we consider the elastic and electric fields due to the disturbed temperature distribution $T_{i}^{(2)}(r, z)(i=1,2)$ only. It is convenient to represent the solutions $u_{z i}(r, z), u_{r i}(r, z)$ and $\phi_{i}(r, z)(i=1,2)$ as the sum of two functions, respectively.

$$
\left.\begin{array}{l}
u_{z i}(r, z)=u_{z i}^{(1)}(r, z)+u_{z i}^{(2)}(r, z), \\
u_{r i}(r, z)=u_{r i}^{(1)}(r, z)+u_{r i}^{(2)}(r, z), \\
\phi_{i}(r, z)=\phi_{i}^{(1)}(r, z)+\phi_{i}^{(2)}(r, z)
\end{array}\right\} \quad(i=1,2)
$$

where $u_{z i}^{(1)}(r, z), u_{r i}^{(1)}(r, z), \phi_{i}^{(1)}(r, z)(i=1,2)$ are the particular solutions of Eqs.(4) replaced $T_{i}$ by $T_{i}^{(2)}$, and $u_{z i}^{(2)}(r, z), u_{r i}^{(2)}(r, z), \phi_{i}^{(2)}(r, z)(i=1,2)$ are the general solutions of 
homogeneous equations obtained by setting $T_{i}=0(i=1,2)$ in Eqs.(4). In the following, the superscripts (1) and (2) indicate the particular and general solutions of Eqs.(4). Substituting Eqs.(29) into Eqs.(1) and (2), one obtains stress $\sigma_{r r i}(r, z), \sigma_{\theta \theta i}(r, z), \sigma_{z z i}(r, z)$, $\sigma_{z r i}(r, z)$ and electric displacement $D_{r i}(r, z), D_{z i}(r, z)(i=1,2)$ expressions.

Using the displacement potential function method (Ueda, 2006a), the particular solutions can be obtained as follows:

$$
\left.\begin{array}{l}
\sigma_{z z i}^{(1)}(r, z)=\sum_{j=1}^{2} \int_{0}^{\infty} p_{1 i j}^{(1)} R_{i j}(s) R_{0}(s) J_{0}(s r) \exp \left(s \tau_{i j} z\right) d s, \\
\sigma_{z r i}^{(1)}(r, z)=\sum_{j=1}^{2} \int_{0}^{\infty} p_{2 i j}^{(1)} R_{i j}(s) R_{0}(s) J_{1}(s r) \exp \left(s \tau_{i j} z\right) d s, \\
D_{z i}^{(1)}(r, z)=\sum_{j=1}^{2} \int_{0}^{\infty} p_{3 i j}^{(1)} R_{i j}(s) R_{0}(s) J_{0}(s r) \exp \left(s \tau_{i j} z\right) d s, \\
u_{z i}^{(1)}(r, z)=\sum_{j=1}^{2} \int_{0}^{\infty} \frac{1}{s} p_{4 i j}^{(1)} R_{i j}(s) R_{0}(s) J_{0}(s r) \exp \left(s \tau_{i j} z\right) d s, \\
u_{r i}^{(1)}(r, z)=\sum_{j=1}^{2} \int_{0}^{\infty} \frac{1}{s} p_{5 i j}^{(1)} R_{i j}(s) R_{0}(s) J_{1}(s r) \exp \left(s \tau_{i j} z\right) d s, \\
\phi_{i}^{(1)}(r, z)=-\sum_{j=1}^{2} \int_{0}^{\infty} \frac{1}{s} p_{6 i j}^{(1)} R_{i j}(s) R_{0}(s) J_{0}(s r) \exp \left(s \tau_{i j} z\right) d s
\end{array}\right\}(i=1,2)
$$

where the constants $p_{k i j}^{(1)}(i, j=1,2, k=1,2, \ldots, 6)$ are given in Appendix A. The general solutions are obtained by using the Hankel transform technique (Sneddon \& Lowengrub, 1969):

$$
\left.\begin{array}{l}
\sigma_{z z i}^{(2)}(r, z)=\sum_{j=1}^{6} \int_{0}^{\infty} s p_{1 i j}^{(2)} A_{i j}(s) J_{0}(s r) \exp \left(s \gamma_{i j} z\right) d s, \\
\sigma_{z r i}^{(2)}(r, z)=\sum_{j=1}^{6} \int_{0}^{\infty} s p_{2 i j}^{(2)} A_{i j}(s) J_{1}(s r) \exp \left(s \gamma_{i j} z\right) d s, \\
D_{z i}^{(2)}(r, z)=\sum_{j=1}^{6} \int_{0}^{\infty} s p_{3 i j}^{(2)} A_{i j}(s) J_{0}(s r) \exp \left(s \gamma_{i j} z\right) d s, \\
u_{z i}^{(2)}(r, z)=\sum_{j=1}^{6} \int_{0}^{\infty} p_{4 i j}^{(2)} A_{i j}(s) J_{0}(s r) \exp \left(s \gamma_{i j} z\right) d s, \\
u_{r i}^{(2)}(r, z)=\sum_{j=1}^{6} \int_{0}^{\infty} p_{5 i j}^{(2)} A_{i j}(s) J_{1}(s r) \exp \left(s \gamma_{i j} z\right) d s, \\
\phi_{i}^{(2)}(r, z)=-\sum_{j=1}^{6} \int_{0}^{\infty} p_{6 i j}^{(2)} A_{i j}(s) J_{0}(s r) \exp \left(s \gamma_{i j} z\right) d s
\end{array}\right\}(i=1,2)
$$

where $A_{i j}(s)(i=1,2, j=1,2, \ldots, 6)$ are the unknown functions to be solved, and the constants $\gamma_{i j}$ and $p_{k i j}^{(2)}(i=1,2, j, k=1,2, \ldots, 6)$ are given in Appendix $\mathrm{B}$. 
Similar to the temperature analysis, the problem may be reduced to a system of singular integral equations by taking the second boundary conditions (7)-(9) into consideration and by defining the following new unknown functions $G_{l}(r)(l=1,2,3)$ :

$$
\begin{gathered}
G_{1}(r)=\left\{\begin{array}{cc}
\frac{\partial}{\partial r}\left\{u_{z 1}^{(2)}(r, 0)-u_{z 2}^{(2)}(r, 0)\right\} & (0 \leq r<c) \\
0 & (c \leq r<\infty)
\end{array}\right\} \\
G_{2}(r)=\left\{\begin{array}{cc}
r \frac{\partial}{\partial r}\left[\frac{1}{r}\left\{u_{r 1}^{(2)}(r, 0)-u_{r 2}^{(2)}(r, 0)\right\}\right] & (0 \leq r<c) \\
0 & (c \leq r<\infty)
\end{array}\right\} \\
G_{3}(r)=\left\{\begin{array}{cc}
-\frac{\partial}{\partial r}\left\{\phi_{1}^{(2)}(r, 0)-\phi_{2}^{(2)}(r, 0)\right\} & (0 \leq r<c) \\
0 & (c \leq r<\infty)
\end{array}\right\}
\end{gathered}
$$

Making use of the first boundary conditions (7)-(9) with Eqs.(10), we have the following system of singular integral equations for the determination of the unknown functions $\mathrm{G}_{l}(t)(l=1,2,3)$ :

$$
\begin{aligned}
& \int_{0}^{c} t\left[\left\{Z_{11}^{\infty} M_{0}^{(1)}(t, r)+M_{11}(t, r)\right\} G_{1}(t)+M_{12}(t, r) G_{2}(t)+\right. \\
& \left.+\left\{Z_{13}^{\infty} M_{0}^{(1)}(t, r)+M_{13}(t, r)\right\} G_{3}(t)\right] d t=\sigma_{z z 0}(r) \quad(0 \leq r<c) \\
& \int_{0}^{c} t\left[M_{21}(t, r) G_{1}(t)+\left\{Z_{22}^{\infty} M_{1}^{(1)}(t, r)+M_{22}(t, r)\right\} G_{2}(t)+\right. \\
& \left.+M_{23}(t, r) G_{3}(t)\right] d t=\sigma_{z r 0}(r) \quad(0 \leq r<c) \\
& \int_{0}^{c} t\left[\left\{Z_{31}^{\infty} M_{0}^{(1)}(t, r)+M_{31}(t, r)\right\} G_{1}(t)+M_{32}(t, r) G_{2}(t)+\right. \\
& \left.+\left\{Z_{33}^{\infty} M_{0}^{(1)}(t, r)+M_{33}(t, r)\right\} G_{3}(t)\right] d t=D_{z 0}(r) \quad(0 \leq r<c)
\end{aligned}
$$

where the kernel functions $M_{1}^{(1)}(t, r), M_{k l}(t, r)$ and the constants $Z_{k l}^{\infty}(k, l=1,2,3)$ are given by

$$
\begin{gathered}
M_{1}^{(1)}(t, r)=\left\{\begin{array}{cc}
\frac{4}{\pi r t}\left\{K\left(\frac{r}{t}\right)-E\left(\frac{r}{t}\right)\right\}+\frac{2}{\pi r t}\left\{\frac{t^{2}}{t^{2}-r^{2}} E\left(\frac{r}{t}\right)-K\left(\frac{r}{t}\right)\right\} & (r<t), \\
\frac{4}{\pi t^{2}}\left\{K\left(\frac{t}{r}\right)-E\left(\frac{t}{r}\right)\right\}+\frac{2}{\pi} \frac{1}{t^{2}-r^{2}} E\left(\frac{t}{r}\right) \quad(r>t)
\end{array}\right\} \\
M_{k l}(t, r)=\left\{\begin{array}{lc}
\int_{0}^{\infty} s\left\{Z_{k l}(s)-Z_{k l}^{\infty}\right\} J_{0}(s r) J_{1}(s t) d s & (k=1,3, l=1,3), \\
\int_{0}^{\infty} s\left\{Z_{k l}(s)-Z_{k l}^{\infty}\right\} J_{0}(s r) J_{2}(s t) d s & (k=1,3, l=2), \\
\int_{0}^{\infty} s\left\{Z_{k l}(s)-Z_{k l}^{\infty}\right\} J_{1}(s r) J_{1}(s t) d s & (k=2, l=1,3), \\
\int_{0}^{\infty} s\left\{Z_{k l}(s)-Z_{k l}^{\infty}\right\} J_{1}(s r) J_{2}(s t) d s & (k=2, l=2)
\end{array}\right\}
\end{gathered}
$$




$$
Z_{k l}(s)=\sum_{j=1}^{6} p_{k 1 j}^{(2)} d_{1 j l}(s), \quad Z_{k l}^{\infty}=\lim _{s \rightarrow \infty} Z_{k l}(s) \quad(k, l=1,2,3)
$$

In Eq.(40), the functions $d_{1 j l}(s)(j=1,2, \ldots, 6, l=1,2,3)$ are given in Appendix C.

The functions $\sigma_{z z 0}(r), \sigma_{z r 0}(r)$ and $D_{z 0}(r)$, which correspond to the stress and electric displacement components induced by the disturbed temperature field $T_{i}^{(2)}(r, z)(i=1,2)$ on the $r$-axis in the plate without crack, are obtained as follows:

$$
\left.\begin{array}{l}
\sigma_{z z 0}(r)=\int_{0}^{\infty} R_{0}(s)\left\{\sum_{j=1}^{6} p_{11 j}^{(2)} d_{1 j}^{T}(s)+\sum_{j=1}^{2} p_{11 j}^{(1)} R_{1 j}(s)\right\} J_{0}(s r) d s, \\
\sigma_{z r 0}(r)=\int_{0}^{\infty} R_{0}(s)\left\{\sum_{j=1}^{6} p_{21 j}^{(2)} d_{1 j}^{T}(s)+\sum_{j=1}^{2} p_{21 j}^{(1)} R_{1 j}(s)\right\} J_{1}(s r) d s, \\
D_{z 0}(r)=\int_{0}^{\infty} R_{0}(s)\left\{\sum_{j=1}^{6} p_{31 j}^{(2)} d_{1 j}^{T}(s)-\sum_{j=1}^{2} p_{31 j}^{(1)} R_{1 j}(s)\right\} J_{0}(s r) d s
\end{array}\right\}
$$

where the functions $d_{1 j}^{T}(s) \quad(j=1,2, \ldots, 6)$ are also given in Appendix C. These components are superficial quantities and have no physical meaning in this analysis. However, they are equivalent to the crack face tractions in solving the crack problem by a proper superposition.

To solve the singular integral equations (21) and (35)- (37) by using the Gauss-Jacobi integration formula (Sih, 1972), we introduce the following functions $\Phi_{l}(t)(l=0,1,2,3)$ :

$$
G_{l}(t)=\left(\frac{c+t}{c-t}\right)^{1 / 2} \Phi_{l}(t) \quad(l=0,1,2,3)
$$

Then the stress intensity factors $K_{\mathrm{I}}, K_{\mathrm{II}}$ and the electric displacement intensity factor $K_{\mathrm{D}}$ may be defined and evaluated as:

$$
\left.\begin{array}{r}
K_{\mathrm{I}}=\lim _{r \rightarrow c^{+}}\{2 \pi(c-r)\}^{1 / 2} \sigma_{z z 1}(r, 0)=(\pi c)^{1 / 2}\left\{Z_{11}^{\infty} \Phi_{1}(c)+Z_{13}^{\infty} \Phi_{3}(c)\right\}, \\
K_{\mathrm{II}}=\lim _{r \rightarrow c^{+}}\{2 \pi(c-r)\}^{1 / 2} \sigma_{z r 1}(r, 0)=(\pi c)^{1 / 2} Z_{22}^{\infty} \Phi_{2}(c), \\
K_{\mathrm{D}}=\lim _{r \rightarrow c^{+}}\{2 \pi(c-r)\}^{1 / 2} D_{z 1}(r, 0)=(\pi c)^{1 / 2}\left\{Z_{31}^{\infty} \Phi_{1}(c)+Z_{33}^{\infty} \Phi_{3}(c)\right\}
\end{array}\right\}
$$

\section{Numerical results and discussion}

For the numerical calculations, the thermo-electro-elastic properties of the plate are assumed to be ones of cadmium selenide with the following properties (Ashida \& Tauchert, 1998).

The values of the coefficients of heat conduction for cadmium selenide could not be found in the literature. Since the values of them for orthotropic Alumina $\left(\mathrm{Al}_{2} \mathrm{O}_{3}\right)$ are $\kappa_{r}=21.25[\mathrm{~W} / \mathrm{mK}]$ and $\kappa_{z}=29.82[\mathrm{~W} / \mathrm{mK}](\mathrm{Dag}, 2006)$, the value $\kappa^{2}=\kappa_{r} / \kappa_{z}=1 / 1.5$ is assumed. To examine the effects of the normalized crack size $c / h$ and the normalized crack 
location $h_{1} / h$ on the stress and electric displacement intensity factors, the solutions of the system of the singular integral equations have been computed numerically.

$$
\begin{array}{rlrl}
c_{11} & =74.1 \times 10^{9}\left[\mathrm{~N} / \mathrm{m}^{2}\right], & & c_{12}=45.2 \times 10^{9}\left[\mathrm{~N} / \mathrm{m}^{2}\right], \\
c_{13} & =39.3 \times 10^{9}\left[\mathrm{~N} / \mathrm{m}^{2}\right], & & c_{33}=83.6 \times 10^{9}\left[\mathrm{~N} / \mathrm{m}^{2}\right], \\
c_{44} & =13.2 \times 10^{9}\left[\mathrm{~N} / \mathrm{m}^{2}\right], & & \\
e_{31}=-0.16\left[\mathrm{C} / \mathrm{m}^{2}\right], & & e_{33}=0.347\left[\mathrm{C} / \mathrm{m}^{2}\right], \\
e_{15}=-0.138\left[\mathrm{C} / \mathrm{m}^{2}\right], & & \\
\varepsilon_{11}=82.6 \times 10^{-12}[\mathrm{C} / \mathrm{Vm}], & \varepsilon_{33}=90.3 \times 10^{-12}[\mathrm{C} / \mathrm{Vm}], \\
\lambda_{11}=0.621 \times 10^{6}\left[\mathrm{~N} / \mathrm{Km}^{2}\right], & \lambda_{33}=0.551 \times 10^{6}\left[\mathrm{~N} / \mathrm{Km}^{2}\right], \\
p_{z}=-2.94 \times 10^{-6}\left[\mathrm{CK}^{-1} \mathrm{~m}^{-2}\right] . &
\end{array}
$$

In the first set of calculations, we consider the temperature field and the electro-elastic fields without crack. Figure 2 shows the normalized temperature $\left(T_{i}(x)-T_{20}\right) / T_{0}(i=1,2)$ on the crack faces $\left(0 \leq r<c, z \rightarrow 0^{ \pm}\right)$and the crack extended line $(c \leq r \leq 2 c, z=0)$ for $h_{1} / h=0.25$ and $c / h=0.5$, where $T_{0}=T_{10}-T_{20}$. The maximum local temperature difference across the crack occurs at the center of the crack.

Figure 3 exhibits the normalized stress components $\left(\sigma_{z z 0}(r), \sigma_{z r 0}(r)\right) / \lambda_{33} T_{0}$ and the electric displacement component $D_{z 0}(r) / p_{z} T_{0}$ on the $r$-axis in the strip without crack due to the temperature shown in Figure 2. The maximum absolute values of $\sigma_{z z 0}(r)$ and $D_{z 0}(r)$ occur at the center of the crack $(r / c=0.0)$, whereas the maximum value of $\sigma_{z r 0}(r)$ occurs at the crack tip $(r / c=1.0)$.

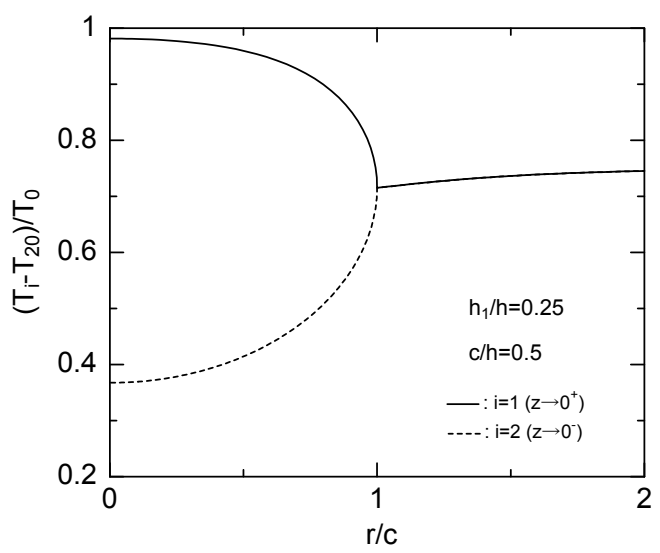

Fig. 2. The temperature on the crack faces and the crack extended line for $c / h=0.5$ and $h_{1} / h=0.25$

In the second set of calculations, we study the influence of the crack size on the stress and electric displacement intensity factors. Figures $4(\mathrm{a})-(\mathrm{c})$ show the plots of the normalized stress and electric displacement intensity factors $\left(K_{\mathrm{I}}, K_{\mathrm{II}}\right) / \lambda_{33} T_{0}(\pi c)^{1 / 2}, K_{\mathrm{D}} / p_{z} T_{0}(\pi c)^{1 / 2}$ versus $c / h$ for $h_{1} / h=0.25,0.5$ and 0.75 . Because of symmetry, the values of $K_{\mathrm{I}}$ and $K_{\mathrm{D}}$ 


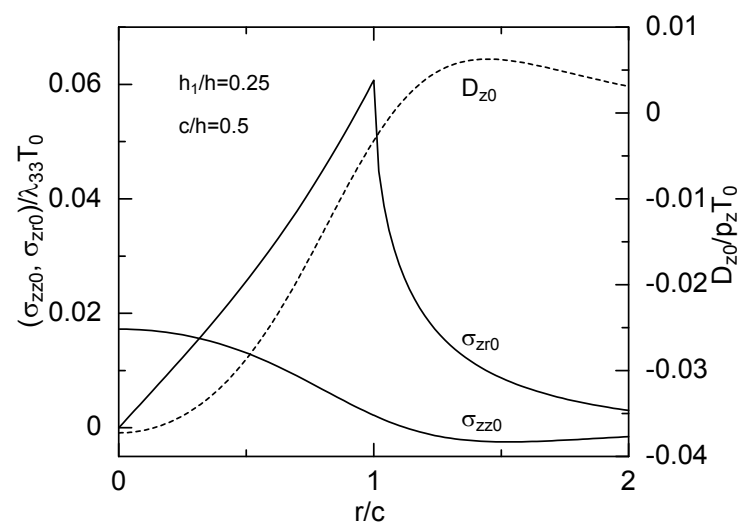

Fig. 3. The stress components $\sigma_{z z 0}, \sigma_{z r 0}$ and the electric displacement component $D_{z 0}$ on the $r$-axis without crack due to the temperature shown in Fig. 2
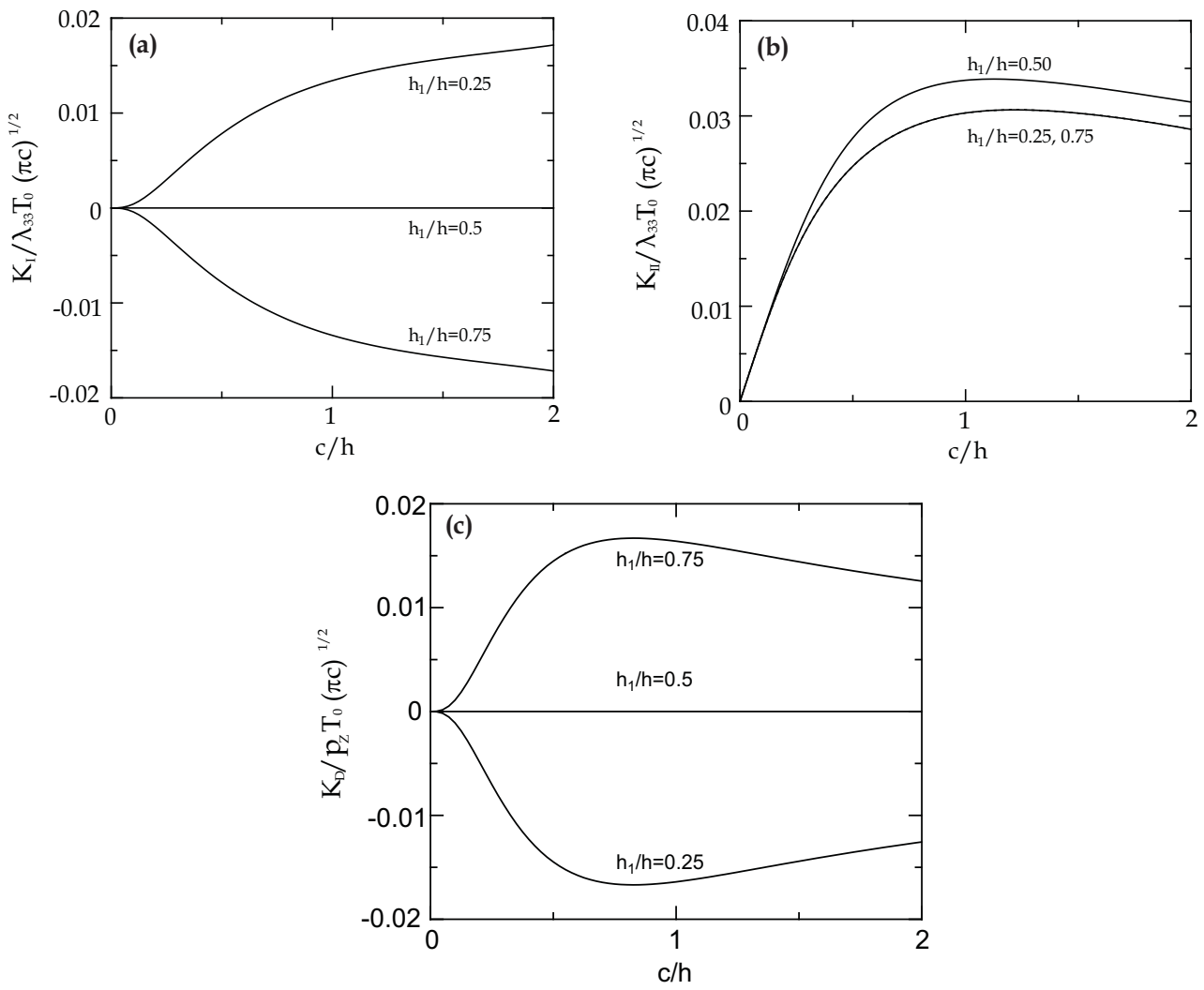

Fig. 4. (a) The effect of the crack size on the stress intensity factor $K_{\mathrm{I}}$. (b) The effect of the crack size on the stress intensity factor $K_{\mathrm{II}}$. (c) The effect of the crack size on the electric displacement intensity factor $K_{D}$ 
for $h_{1} / h=0.5$ are zero, and $\left[K_{\mathrm{I}}\right]_{h_{1} / h=0.25}=-\left[K_{\mathrm{I}}\right]_{h_{1} / h=0.75}, \quad\left[K_{\mathrm{II}}\right]_{h_{1} / h=0.25}=\left[K_{\mathrm{II}}\right]_{h_{1} / h=0.75}$ $\left[K_{\mathrm{D}}\right]_{h_{1} / h=0.25}=-\left[K_{\mathrm{D}}\right]_{h_{1} / h=0.75}$. The absolute value of $K_{\mathrm{I}} / \lambda_{33} T_{0}(\pi c)^{1 / 2}$ for $h_{1} / h=0.25$ and 0.75 monotonically increases with increasing $c / h$, but the value of $K_{\mathrm{II}} / \lambda_{33} T_{0}(\pi c)^{1 / 2}$ and the absolute value of $K_{\mathrm{D}} / p_{z} T_{0}(\pi c)^{1 / 2}$ increase at first, reach maximum values and then decrease with increasing $c / h$. The value of $K_{\mathrm{I}}$ for $h_{1} / h=0.75$ becomes negative so that the contact of the crack faces would occur. The results presented here without considering this effect may not be exact but would be more conservative. Since the contact of the crack faces will increase the friction between the faces and make thermo-electrical transfer across the crack faces easier, the stress and electric displacement intensity factors would be lowered by these two factors.

In the final set of calculations, we investigate the influence of the crack location on the intensity factors. Figure 5 indicates the effect of the crack location on $K_{\mathrm{I}}, K_{\mathrm{II}}$ and $K_{\mathrm{D}}$ for $c / h=0.5$. As $h_{1} / h$ increases, the values of $K_{\mathrm{I}}$ and $K_{\mathrm{D}}$ tend to decrease or increase monotonically. The value of $K_{\mathrm{II}} / \lambda_{33} T_{0}(\pi c)^{1 / 2}$ decreases if the crack approaches the free boundaries $\left(h_{1} / h \rightarrow 0.0\right.$ or 1.0$)$, and the peak value of $K_{\mathrm{II}} / \lambda_{33} T_{0}(\pi c)^{1 / 2}=0.0277$ occurs at $h_{1} / h=0.5$.

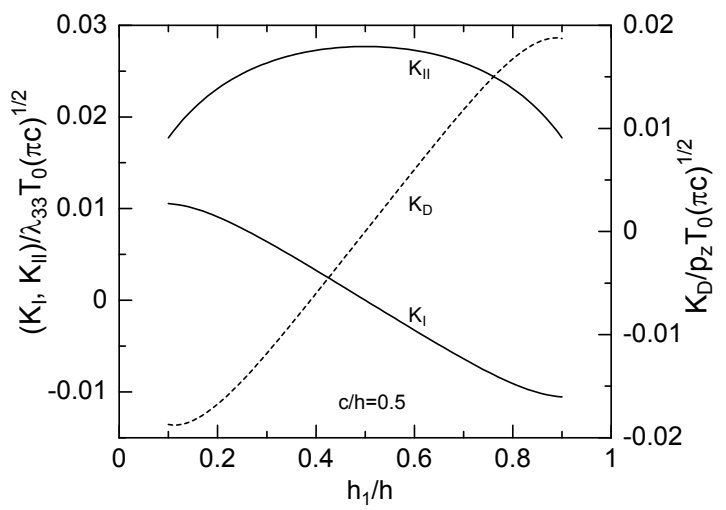

Fig. 5 The effect of the crack location on the stress intensity factors $K_{\mathrm{I}}, K_{\mathrm{II}}$ and the electric displacement intensity factor $K_{D}$

\section{Conclusion}

An example of the application of Hankel transform for solving a mixed-mode thermoelectro-elastic fracture problem of a piezoelectric material strip with a parallel penny-shaped crack is explained. The effects of the crack size $(c / h)$ and the crack location $\left(h_{1} / h\right)$ on the fracture behavior are analyzed. The following facts can be found from the numerical results.

1. The large shear stress occurs in the strip without crack due to the disturbed temperature field.

2. The normalized intensity factors are under the great influence of the geometric parameters $h_{1} / h$ and $c / h$. 
3. For the case of $h_{1} / h>0.5$, mode I stress intensity factor becomes negative so that the contact of the crack faces would occur.

4. The intensity factors of crack near the free surfaces due to the thermal load are not so large.

\section{Appendix A}

The constants $p_{k i j}^{(1)}(i, j=1,2, k=1,2, \ldots, 6)$ are

$$
\left.\begin{array}{c}
p_{1 i j}^{(1)}=\left(c_{13}-c_{33} k_{i j} \tau_{i j}^{2}\right) C_{i j}-e_{33} \tau_{i j} N_{i j}-\lambda_{33}, \\
p_{2 i j}^{(1)}=c_{44}\left(1+k_{i j}\right) \tau_{i j} C_{i j}+e_{15} N_{i j}, \\
p_{3 i j}^{(1)}=\left(e_{31}-e_{33} k_{i j} \tau_{i j}^{2}\right) C_{i j}+\varepsilon_{33} \tau_{i j} N_{i j}+p_{z}, \\
p_{4 i j}^{(1)}=-k_{i j} \tau_{i j} C_{i j}, p_{5 i j}^{(1)}=C_{i j}, p_{6 i j}^{(1)}=N_{i j}
\end{array}\right\}
$$

where

$$
\begin{aligned}
& C_{i j}=\frac{b_{21}^{\prime}-b_{22}^{\prime} \tau_{i j}^{2}}{m_{i j}^{(1)}+k_{i j} m_{i j}^{(2)}}, \\
& \left.N_{i j}=\frac{n_{i j}^{(11)}+k_{i j} n_{i j}^{(12)}}{m_{i j}^{(1)}+k_{i j} m_{i j}^{(2)}},\right\} \quad(i, j=1,2) \\
& k_{i j}=-\frac{\tau_{i j}^{2} n_{i j}^{(11)}+n_{i j}^{(21)}}{\tau_{i j}^{2} n_{i j}^{(12)}+n_{i j}^{(22)}}
\end{aligned}
$$

with

$$
\begin{aligned}
& m_{i j}^{(1)}=a^{\prime}{ }_{41}-a^{\prime}{ }_{42} \tau_{i j}^{2}, \\
& m_{i j}^{(2)}=\left(a^{\prime}{ }_{44} \tau_{i j}^{2}-a^{\prime}{ }_{43}\right) \tau_{i j}^{2} \text {, } \\
& n_{i j}^{(11)}=\left\{\left(H_{1 r}+H_{3 r}\right)\left(b^{\prime}{ }_{21}-b^{\prime}{ }_{22} \tau_{i j}^{2}\right)+H_{4 r} m_{i j}^{(1)}\right\} \tau_{i j}, \\
& n_{i j}^{(21)}=\left\{\left(H_{1 z}+H_{3 z}\right)\left(b^{\prime}{ }_{21}-b^{\prime}{ }_{22} \tau_{i j}^{2}\right)+H_{4 z} m_{i j}^{(1)}\right\} \tau_{i j} \text {, } \\
& n_{i j}^{(12)}=\left\{\left(H_{1 r}-H_{2 r} \tau_{i j}^{2}\right)\left(b^{\prime}{ }_{21}-b^{\prime}{ }_{22} \tau_{i j}^{2}\right)+H_{4 r} m_{i j}^{(2)}\right\} \tau_{i j} \text {, } \\
& n_{i j}^{(22)}=\left\{\left(H_{1 z}-H_{2 z} \tau_{i j}^{2}\right)\left(b^{\prime}{ }_{21}-b^{\prime}{ }_{22} \tau_{i j}^{2}\right)+H_{4 z} m_{i j}^{(2)}\right\} \tau_{i j} \\
& (i, j=1,2) \\
& H_{1 r}=\frac{c_{44} \varepsilon_{33}+e_{15} e_{33}}{e_{33} \varepsilon_{11}-e_{15} \varepsilon_{33}}, \quad H_{1 z}=\frac{c_{44} \varepsilon_{11}+e_{15}^{2}}{e_{15} \varepsilon_{33}-e_{33} \varepsilon_{11}}, \quad H_{2 r}=\frac{c_{33} \varepsilon_{33}+e_{33}^{2}}{e_{33} \varepsilon_{11}-e_{15} \varepsilon_{33}}, \quad H_{2 z}=\frac{c_{33} \varepsilon_{11}+e_{15} e_{33}}{e_{15} \varepsilon_{33}-e_{33} \varepsilon_{11}}, \\
& H_{3 r}=\frac{c_{13} \varepsilon_{33}+e_{31} e_{33}}{e_{33} \varepsilon_{11}-e_{15} \varepsilon_{33}}, \quad H_{3 z}=\frac{c_{13} \varepsilon_{11}+e_{15} e_{31}}{e_{15} \varepsilon_{33}-e_{33} \varepsilon_{11}}, \quad H_{4 r}=\frac{p_{z} e_{33}-\lambda_{33} \varepsilon_{33}}{e_{33} \varepsilon_{11}-e_{15} \varepsilon_{33}}, \quad H_{4 z}=\frac{p_{z} e_{15}-\lambda_{33} \varepsilon_{11}}{e_{15} \varepsilon_{33}-e_{33} \varepsilon_{11}}
\end{aligned}
$$




$$
\left.\begin{array}{ll}
a_{41}^{\prime}=c_{11}, & a_{42}^{\prime}=c_{44}+\left(e_{15}+e_{31}\right)\left(H_{1 r}+H_{3 r}\right) \\
a_{43}^{\prime}=c_{13}+c_{44}+\left(e_{15}+e_{31}\right) H_{1 r}, & a_{44}^{\prime}=\left(e_{15}+e_{31}\right) H_{2 r}, \\
b_{21}^{\prime}=\lambda_{11}, & b_{22}^{\prime}=-\left(e_{15}+e_{31}\right) H_{4 r}
\end{array}\right\}
$$

\section{Appendix B}

The constants $\gamma_{i j}(i=1,2, j=1,2, \ldots, 6)$ are the roots of the following characteristic equations:

$$
\begin{aligned}
& \left(f_{4} g_{2}^{\prime}+g_{4} f_{2}^{\prime}\right) \gamma_{i j}^{6}+\left(f_{4} g_{0}^{\prime}+f_{2} g_{2}^{\prime}+g_{4} f_{0}^{\prime}+g_{2} f_{2}^{\prime}\right) \gamma_{i j}^{4}+ \\
& +\left(f_{2} g_{0}^{\prime}+f_{0} g_{2}^{\prime}+g_{2} f_{0}^{\prime}+g_{0} f_{2}^{\prime}\right) \gamma_{j}^{2}+\left(f_{0} g_{0}^{\prime}+g_{0} f_{0}^{\prime}\right)=0
\end{aligned} \quad(i=1,2, j=1,2, \ldots, 6)
$$

where $\mathfrak{R}\left[\gamma_{1 j}\right]<\mathfrak{R}\left[\gamma_{1 j+1}\right], \mathfrak{R}\left[\gamma_{2 j}\right]>\mathfrak{R}\left[\gamma_{2 j+1}\right](j=1,2, \ldots, 5)$ and

$$
\left.\begin{array}{rl}
f_{4}= & c_{44} e_{33}, \\
f_{2}= & \left(c_{13}+c_{44}\right)\left(e_{15}+e_{31}\right)-c_{11} e_{33}-c_{44} e_{15}, \\
f_{0}= & c_{11} e_{15}, \\
f_{2}^{\prime}= & c_{33}\left(e_{15}+e_{31}\right)-e_{33}\left(c_{13}+c_{44}\right), \\
f_{0}^{\prime}=-c_{44}\left(e_{15}+e_{31}\right)+e_{15}\left(c_{13}+c_{44}\right) \\
\\
g_{4}=c_{44} \varepsilon_{33}, \\
g_{2}=-\left(e_{15}+e_{31}\right)^{2}-c_{11} \varepsilon_{33}-c_{44} \varepsilon_{11}, \\
g_{0}=c_{11} \varepsilon_{11}, \\
g_{2}^{\prime}=e_{33}\left(e_{15}+e_{31}\right)+\varepsilon_{33}\left(c_{13}+c_{44}\right), \\
g_{0}^{\prime}=-e_{15}\left(e_{15}+e_{31}\right)-\varepsilon_{11}\left(c_{13}+c_{44}\right)
\end{array}\right\}
$$

The functions $p_{k i j}^{(2)}(s)(i=1,2, j, k=1,2, \ldots, 6)$ are

$$
\left.\begin{array}{c}
p_{i i j}^{(2)}=c_{13} a_{i j}+\gamma_{i j}\left(c_{33}-e_{33} b_{i j}\right), \\
p_{2 i j}^{(2)}=c_{44}\left(\gamma_{i j} a_{i j}-1\right)+e_{15} b_{i j}, \\
p_{3 i j}^{(2)}=e_{31} a_{i j}+\gamma_{i j}\left(e_{310}+\varepsilon_{330} b_{i j}\right), \\
p_{4 i j}^{(2)}=1, \\
p_{5 i j}^{(2)}=a_{i j}, \\
p_{6 i j}^{(2)}=b_{i j}
\end{array}\right\}
$$

where $a_{i j}$ and $b_{i j}(i=1,2, j=1,2, \ldots, 6)$ are given by

$$
\left.\begin{array}{c}
a_{i j}=\frac{g_{2}^{\prime} \gamma_{i j}^{2}+g_{0}^{\prime}}{g_{4} \gamma_{i j}^{4}+g_{2} \gamma_{i j}^{2}+g_{0}}, \\
e_{i j}=-\frac{\left(c_{44} \gamma_{i j}^{2}-c_{11}\right) a_{i j}-c_{13}-c_{44}}{e_{15}+e_{31}}
\end{array}\right\} \quad(i=1,2, j=1,2, \ldots, 6)
$$




\section{Appendix C}

The functions $d_{i j k}(s)(i=1,2, j=1,2, \ldots, 6, k=1,2,3)$ are given by

$$
\left.\begin{array}{l}
d_{1 j k}(s)=q_{j, k+9}(s), \\
d_{2 j k}(s)=q_{j+6, k+9}(s)
\end{array}\right\} \quad(j=1,2, \ldots, 6, k=1,2,3)
$$

where the functions $q_{j, k}(s)(j, k=1,2, \ldots, 12)$ are the elements of a square matrix $Q=\Delta^{-1}$ of order 12. The elements $\delta_{j, k}(s)(j, k=1,2, \ldots, 12)$ of the square matrix $\Delta$ are given by

$$
\begin{aligned}
& \delta_{j, k}(s)=p_{j 1 k}^{(2)} \exp \left(s \gamma_{1 k} h_{1}\right) \quad(j=1,2,3), \\
& \left.\begin{array}{cr}
\delta_{j+3, k+6}(s)=p_{j 2 k}^{(2)} \exp \left(-s \gamma_{2 k} h_{2}\right) & (j=1,2,3), \\
\delta_{j+6, k}(s)=p_{j 1 k}^{(2)} & (j=1,2, \ldots, 6),
\end{array}\right\}(k=1,2, \ldots, 6) \\
& \delta_{j+6, k+6}(s)=-p_{j 2 k}^{(2)} \quad(j=1,2, \ldots, 6)
\end{aligned}
$$

The functions $d_{i j}^{T}(s)(i=1,2, j=1,2, \ldots, 6)$ are

$$
\left.\begin{array}{l}
d_{1 j}^{T}(s)=\sum_{k=1}^{12} q_{j, k}(s) u_{k}(s), \\
d_{2 j}^{T}(s)=\sum_{k=1}^{12} q_{j+6, k}(s) u_{k}(s)
\end{array}\right\}(j=1,2, \ldots, 6)
$$

where

$$
\left.\begin{array}{cc}
u_{k}(s)=-\frac{R_{0}(s)}{s} \sum_{j=1}^{2} p_{k 1 j}^{(1)} R_{1 j}(s) \exp \left(s \tau_{1 j} h_{1}\right) & (k=1,2,3), \\
u_{k+3}(s)=-\frac{R_{0}(s)}{s} \sum_{j=1}^{2} p_{k 2 j}^{(1)} R_{2 j}(s) \exp \left(-s \tau_{2 j} h_{2}\right) & (k=1,2,3), \\
u_{k+6}(s)=-\frac{R_{0}(s)}{s} \sum_{j=1}^{2}\left\{p_{k 1 j}^{(1)} R_{1 j}(s)-p_{k 2 j}^{(1)} R_{2 j}(s)\right\} & (k=1,2, \ldots, 6)
\end{array}\right\}
$$

\section{References}

Ashida, F. \& Tauchert, T.R. (1998). Transient Response of a Piezothermoelastic Circular Disk under Axisymmetric Heating. Acta Mechanica, Vol. 128, pp. 1-14, 0001-5970

Dag, S., Ilhan, K.A. \& Erdogan, F. (2006), Mixed-Mode Stress Intensity Factors for an Embedded Crack in an Orthotropic FGM Coating, Proceedings of the International Conference FGM IX, 978-0-7354-0492-2, Oahu Island, Hawaii, October 2006

Erdogan, F. \& Wu, B.H. (1996). Crack Problems in FGM Layers under Thermal Stresses. Journal of Thermal Stresses, Vol. 19, pp. 237-265, 0149-5739 
Rao, S.S. \& Sunar, M. (1994). Piezoelectricity and Its Use in Disturbance Sensing and Control of Flexible Structures: a Survey. Applied Mechanics Review, Vol. 47, pp. 113-123, 0003-6900

Sih, G.C. (Ed.). (1972). Methods of Analysis and Solution of Crack Problems, Noordhoff, International Publishing, 978-9048182466, Leyden

Sneddon, I.N. \& Lowengrub, M. (1969). Crack Problems in the Classical Theory of Elasticity, John Wiley \& Sons, Inc., 978-0471808459, New York

Tauchert, T.R. (1992). Piezothermoelastic Behavior of a Laminated Plate. Journal of Thermal Stresses, Vol. 15, pp. 25-37, 0149-5739

Ueda, S. (2006a). The Crack Problem in Piezoelectric Strip under Thermoelectric Loading. Journal of Thermal Stresses, Vol. 29, pp. 295-316, 0149-5739

Ueda, S. (2006b). Thermal Stress Intensity Factors for a Normal Crack in a Piezoelectric Strip. Journal of Thermal Stresses, Vol. 29, pp. 1107-1126, 0149-5739 


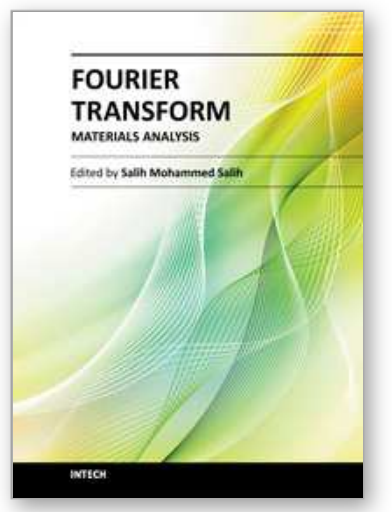

\author{
Fourier Transform - Materials Analysis \\ Edited by Dr Salih Salih
}

ISBN 978-953-51-0594-7

Hard cover, 260 pages

Publisher InTech

Published online 23, May, 2012

Published in print edition May, 2012

The field of material analysis has seen explosive growth during the past decades. Almost all the textbooks on materials analysis have a section devoted to the Fourier transform theory. For this reason, the book focuses on the material analysis based on Fourier transform theory. The book chapters are related to FTIR and the other methods used for analyzing different types of materials. It is hoped that this book will provide the background, reference and incentive to encourage further research and results in this area as well as provide tools for practical applications. It provides an applications-oriented approach to materials analysis written primarily for physicist, Chemists, Agriculturalists, Electrical Engineers, Mechanical Engineers, Signal Processing Engineers, and the Academic Researchers and for the Graduate Students who will also find it useful as a reference for their research activities.

\title{
How to reference
}

In order to correctly reference this scholarly work, feel free to copy and paste the following:

Sei Ueda (2012). Application of Hankel Transform for Solving a Fracture Problem of a Cracked Piezoelectric Strip Under Thermal Loading, Fourier Transform - Materials Analysis, Dr Salih Salih (Ed.), ISBN: 978-953-510594-7, InTech, Available from: http://www.intechopen.com/books/fourier-transform-materialsanalysis/application-of-hankel-transform-for-solving-a-fracture-problem-of-a-cracked-piezoelectric-strip-unde

\section{INTECH}

open science | open minds

\author{
InTech Europe \\ University Campus STeP Ri \\ Slavka Krautzeka 83/A \\ 51000 Rijeka, Croatia \\ Phone: +385 (51) 770447 \\ Fax: +385 (51) 686166 \\ www.intechopen.com
}

\author{
InTech China \\ Unit 405, Office Block, Hotel Equatorial Shanghai \\ No.65, Yan An Road (West), Shanghai, 200040, China \\ 中国上海市延安西路65号上海国际贵都大饭店办公楼 405 单元 \\ Phone: +86-21-62489820 \\ Fax: +86-21-62489821
}


(C) 2012 The Author(s). Licensee IntechOpen. This is an open access article distributed under the terms of the Creative Commons Attribution 3.0 License, which permits unrestricted use, distribution, and reproduction in any medium, provided the original work is properly cited. 